\title{
Transient Transfection of the Human Myeloid Cell Line Mono Mac 6 Using Electroporation
}

\author{
BioTechniques 34:142-147 (January 2003)
}

\author{
Niko Klan and Dieter \\ Steinhilber \\ University of Frankfurt, \\ Frankfurt, Germany
}

\section{INTRODUCTION}

Studies of the control of gene expression in eukaryotes strongly rely on the transfer of cloned genes into mammalian cells. The traditional method of gene transfer by the uptake of calcium phosphate/DNA co-precipitates and lipofection works well with fibroblasts and HeLa cells but is much less successful with myeloid cells and myeloid/monocytic cell lines $(19,20)$. Electroporation is a frequently used method for introducing macromolecules and cloned genes into cells that has substantial benefits compared to alternative techniques $(30,31)$. However, transfection efficiency can vary substantially between various cell types. In general, the larger the diameter of the cell, the lower the voltage and capacitance that will be tolerated by the cells before they suffer unacceptable levels of cell death (general killing rates are between $40 \%$ and $90 \%$ ). Increasing the number of cells and the amount of DNA used in the electroporation for studying transient gene expression can circumvent the problems of low transfection efficiency and low promoter/enhancer efficiency. Optimization parameters such as voltage, capacitance, pulse time, composition, and resistance of electroporation media, state of cell growth before electroporation, concentration of DNA, temperature, cell density, and time of assay after transfection (19) are important to obtain acceptable transfection efficiencies. Using the lu- ciferase reporter gene driven by the 5 lipoxygenase core promoter, we have established a transient transfection system that can easily be used to study gene regulation in the Mono Mac 6 cell line. The 5-lipoxygenase gene expression is upregulated during myeloid cell differentiation $(2,3)$. Characterization of the human 5-lipoxygenase promoter revealed that a part of the promoter sequence (from -114 to +9 from the transcriptional start site) is essential for the transcription of 5-lipoxygenase reporter gene constructs in both $\mathrm{HeLa}$ and HL-60 cells (13). Several features of the promoter region such as the lack of TATAA or CCAAT boxes and repeated $\mathrm{G}+\mathrm{C}$-rich elements are similar to housekeeping genes. Previous data suggest that the transcription factors Egr-1 and/or Sp1 are required for basal 5-lipoxygenase transcription $(14,27)$ and that they interact with the 5-lipoxygenase promoter and activate it via repeated response elements located between positions -147 and -23 relative to the transcriptional start site $(13,22,23)$. Interestingly, naturally occurring mutations were found in the 5-lipoxygenase promoter, consisting of the deletion of one or two Sp1-binding sites or the addition of one Sp1-binding site (14). These mutations only slightly alter 5 lipoxygenase promoter activity in reporter gene assays but have a significant impact on the response of asthma patients to 5-lipoxygenase inhibitors (7). Recent data obtained with human myeloid cell lines indicate that the 5- 
lipoxygenase promoter is regulated by the DNA methylation of the $\mathrm{G}+\mathrm{C}$-rich core promoter region $(24,25)$. To study the mechanisms of 5-lipoxygenase promoter regulation, it was important to establish a transient transfection protocol for the 5-lipoxygenase-positive Mono Mac 6 cells.

\section{MATERIALS AND METHODS}

\section{Reagents}

Molecular biology reagents were from MBI Fermentas, Sigma, Invitrogen, Promega, or other sources as indicated in the text. Insulin was a gift from Aventis (Frankfurt, Germany). Nucleospin ${ }^{\circledR}$ Extract columns for direct purification of plasmid DNA (pDNA) were from Macherey-Nagel (Düren, Germany).

\section{Cell Culture}

Mono Mac 6 cells were kindly provided by Dr. H.W.L. Ziegler-Heitbrock (Munich, Germany) (28,29). Cells were grown at $37^{\circ} \mathrm{C}$ in a humidified atmosphere with $6 \% \mathrm{CO}_{2}$ in RPMI 1640 medium supplemented with $10 \%(\mathrm{v} / \mathrm{v})$ FCS, streptomycin $(100 \mu \mathrm{g} / \mathrm{mL})$, and penicillin $(100 \mathrm{U} /$ $\mathrm{mL}), 1 \times$ nonessential amino acids, sodium pyruvate $(1 \mathrm{mM})$, oxalacetate $(1 \mathrm{mM})$, and insulin $(10 \mu \mathrm{g} / \mathrm{mL})$.

\section{Plasmid Constructs}

Vector pN10 was obtained by the insertion of the 5-lipoxygenase promoter fragment from -778 to +53 relative to the transcription start site into the promoterless luciferase reporter vector pGL3Basic (Promega, Mannheim, Germany). Plasmid constructs were checked by DNA sequencing.

\section{Transfections}

Forty-eight hours before transfection, Mono Mac 6 cells were split to $2 \times$ $10^{5}$ cells/mL in RPMI 1640 supplemented with $10 \%$ FCS, streptomycin $(100 \mu \mathrm{g} / \mathrm{mL})$, penicillin $(100 \mathrm{U} / \mathrm{mL})$, $1 \times$ nonessential amino acids, sodium pyruvate $(1 \mathrm{mM})$, oxalacetate $(1 \mathrm{mM})$, and insulin $(10 \mu \mathrm{g} / \mathrm{mL})$, and the cells were cultured at $37^{\circ} \mathrm{C}$ in a humidified atmosphere with $6 \% \mathrm{CO}_{2}$. For transfection, the cells were harvested by centrifugation at $1200 \times g$ for $5 \mathrm{~min}$ at room temperature and washed twice at room temperature with RPMI 1640 that contained neither FCS nor L-glutamine. The cells were then resuspended at a density of $46 \times 10^{6}$ cells $/ \mathrm{mL}$ in RPMI 1640 , and the cell suspension $(0.3 \mathrm{~mL})$ was placed into a $0.4-\mathrm{cm}$ electroporation cuvette. Forty micrograms of supercoiled pDNA were dissolved in water to a final volume of $30 \mu \mathrm{L}$ and added to the cell suspension in the cuvette. The cells and plasmid were incubated for $5 \mathrm{~min}$ at room temperature before electroporation at $975 \mu \mathrm{F}$ and 200 V (Bio-rad ${ }^{\circledR}$ Gene Pulser II) or 200-800 V (Multiporator ${ }^{\circledR}$, Eppendorf, Hamburg, Germany), both of which use exponential decay pulse systems. Immediately after the pulse, the cuvettes were transferred into an ice bath for 20 min. The cells were then transferred to $10 \mathrm{~mL}$ RPMI 1640 containing 10\% FCS, insulin, glutamine, and MEM. Six hours after transfection, the cells were washed once in PBS, $\mathrm{pH} 7.4,0.5 \mathrm{mM}$ $\mathrm{MgCl}_{2}, 0.5 \mathrm{mM} \mathrm{CaCl}$, and lysed in $100 \mu \mathrm{L}$ lysis buffer (Constant Light Signal kit; Roche Applied Science, Mannheim, Germany).

\section{Reporter Gene Assays}

The lysates were assayed for luciferase activity by measuring the light emission in a Microlumat Plus LB96V EG\&G Berthold Luminometer using the Constant Light Signal kit. The luminometer was programmed to integrate light emission for $5 \mathrm{~s}$. pCMVSEAP was used as internal standard, and luciferase activity was normalized to secreted alkaline phosphatase (SEAP) activity.

\section{SEAP Activity Assay}

SEAP activity was determined with the Phospha-Light ${ }^{\mathrm{TM}}$ Secreted Alkaline Phosphatase Reporter Gene Assay System kit (Tropix, Bedford, MA, USA), and assays were performed according to the manufacturer's instructions using $33 \mu \mathrm{L}$ conditioned medium of the transfected cells and $100 \mu \mathrm{L}$ dilution buffer (SEAP kit). Signals were detected with the luminometer that had been pro- grammed to integrate light emission for $1 \mathrm{~s}$. Generally, SEAP activity was determined $6 \mathrm{~h}$ after transfection.

\section{Trypan Blue Stain}

The survival of harvested cells was determined by Trypan Blue exclusion. Forty microliters of Trypan Blue $(0.4 \%$; Sigma Chemical, Deisenhofen, Germany) were added to $40 \mu \mathrm{L}$ cell suspension, and the cells were counted after incubation for $5 \mathrm{~min}$ at room temperature.

\section{Calculation}

Transcriptional activity of pN10 over pGL3Basic (see Figure 3A) was calculated by first subtracting the background mean light unit of mock-transfected cells from the average light unit obtained with the reporter gene luciferase construct for both luciferase and SEAP values, respectively. The corrected light units were then divided by the mean of the light units obtained from pGL3Basic-transfected cells to determine the relative luciferase activity over pGL3Basic as a promoterless vector (16).

When the 5-lipoxygenase promoter activity was determined, the luciferase values were corrected for differences in transfection efficiency by normalizing the luciferase values to the respective value for SEAP, thereby obtaining the relative light units. SEAP values (see Figure 3B) are calculated by subtracting the background mean light unit of mock-transfected cells from the average light unit obtained with the pCMVSEAP construct.

\section{RESULTS}

\section{The Field Strength}

The field strength $(\mathrm{V} / \mathrm{cm})$ of the electrical pulse is an important factor for the cellular survival and transfection efficiency of the cells. If the field strength of the pulse exceeds a characteristic cell-type-specific value, then reversible permeation of the cell membrane occurs. The required voltage depends on the cell diameter and temperature at which electroporation is performed. For Mono Mac 6 cells, which have a diameter of approximate- 
ly $30 \mu \mathrm{m}$, a theoretically expected critical field strength of $444 \mathrm{~V} / \mathrm{cm}$ can be calculated, which corresponds to a minimum pulse voltage of $180 \mathrm{~V}$ if one uses a $0.4-\mathrm{cm}$ cuvette at room temperature. In addition to these parameters, the end volume of the cell suspension in the cuvette must be taken into account. Increasing the pulse voltage can increase the transfection rate but, at the same time, usually also enhances cell mortality. Frequently, killing rates of approximately $50 \%$ are observed under experimental conditions that lead to high transfection efficiencies $(1,4,6)$. Figure 1 shows that a stepwise increase in voltage leads to a decrease in cell survival, which indicates a clear relationship between both parameters. When $40 \mu \mathrm{g}$ 5LO-promoter luciferase reporter gene, $40 \mu \mathrm{g}$ promoterless vector pDNA (pGL3Basic), and $1 \mu \mathrm{g}$ pDNA of the pCMVSEAP construct as a control were transfected at $975 \mu \mathrm{F}$ and the indicated voltages from 50 to $350 \mathrm{~V}$ (Figure 2), reporter gene activity was maximal at $200 \mathrm{~V}$, which is slightly higher than the calculated minimum pulse voltage of $180 \mathrm{~V}$. Figure 2 shows that differences of $50 \mathrm{~V}$ can decrease reporter gene activity to under $10 \%$ of the maximum expression values. These results demonstrate the importance of the optimization of transfection conditions with respect to the transfected cell type $(12,15,21)$.

Thus, using the reporter gene con- structs with the luciferase reporter gene driven by the human 5-lipoxygenase promoter and the secreted alkaline phosphatase reporter gene driven by the CMV promoter, the voltage of 200 was optimal. When voltages higher than 200 were used, reduced reporter gene activities were obtained.

All transfections were performed at room temperature because the survival of the cells was markedly decreased if electroporations were done at $0^{\circ} \mathrm{C}$ or if pre-chilled media were used (data not shown and Reference 17).

\section{Length of the Field Pulse}

In addition to the field strength, a crucial factor for successful transfection is the pulse length (26). The length of the pulse required for efficient transfection is primarily dependent on the diameter of the cell. The larger the cell, the longer the pulse that is necessary for the permeation of the membrane. Obviously, for the electroporation of Mono Mac 6 cells, a pulse length (time constant) of more than $400 \mu$ s seems to be required because when Mono Mac 6 cells were electroporated with the multiporator using pulse lengths $40-400 \mu$ s and a cytomegalovirus (CMV) promoter-driven luciferase reporter gene construct, higher voltages of up to $800 \mathrm{~V}$ were necessary to obtain sufficient luciferase activity. These luciferase activities were still 80 -fold less than the reporter gene activ- ities obtained with $200 \mathrm{~V}$ and $975 \mu \mathrm{F}$ using the Gene Pulser II, which leads to time constants from $\tau=33-39 \mathrm{~ms}$. $\tau$ describes the decay time for the pulse required to decrease to $\mathrm{V}_{\mathrm{o}}$ /e (i.e., about $37 \%$ of $V_{o}$ ) (11). After the transfection of the Mono Mac 6 cells according to our protocol, luciferase activities of the CMV promoter-driven reporter gene construct were up to 100 -fold higher than the values of the 5-lipoxygenase promoter luciferase reporter gene construct, indicating that the CMV promoter is a much more active promoter than that of 5-lipoxygenase.

\section{Influence of pDNA}

The transfection efficiency of electroporation can be affected by the concentration and purity of the pDNA. Therefore, transfections were performed with pDNA purified with $\mathrm{Nu}$ cleospin Extract columns, and the purity was checked by UV spectroscopy $\left(\mathrm{A}_{260} / 280 \geq 1.8\right)(8,10)$. Mono Mac 6 cells were transfected with $4,20,40$, and $80 \mu \mathrm{g} 5$-lipoxygenase promoter luciferase plasmid at $200 \mathrm{~V}$ and $975 \mu \mathrm{F}$, and the luciferase activity was determined $6 \mathrm{~h}$ after transfection (Figure 3 ). Luciferase activity increased with the amount of the transfected plasmid up to $40 \mu \mathrm{g}$, resulting in approximately 100 fold higher reporter gene activity compared to the promoterless vector (pGL3Basic) (Figure 3A). SEAP activ-

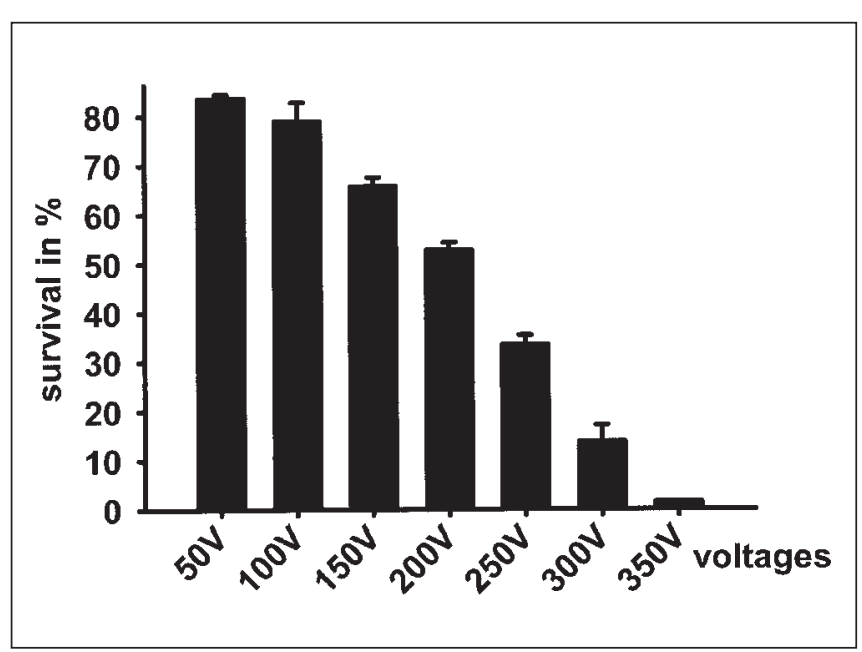

Figure 1. Mean survival of Mono Mac 6 cells after electroporation. Cells were transfected at the indicated voltages, and the survival of the cells was determined by Trypan Blue exclusion $6 \mathrm{~h}$ after electroporation. Transfections were performed in triplicate.

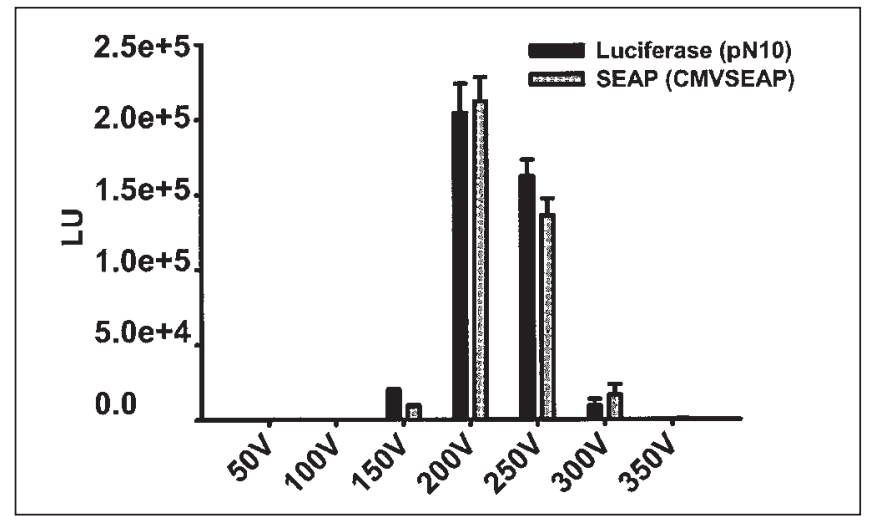

Figure 2. Relation between voltage and reporter gene activities of transiently transfected Mono Mac 6 cells using electroporation. Forty micrograms of the 5-lipoxygenase promoter luciferase reporter gene construct pN10 and $1 \mu \mathrm{g}$ CMV promoter-driven SEAP reporter gene were transfected at the indicated voltages. Six hours after electroporation, the cells were harvested, and reporter gene assays were performed. Results are indicated as the mean value of the corrected light units and the SEM. LU, light units. Transfections were performed in triplicate. 
ity was highest at $20 \mu \mathrm{g}$ N10 plasmid DNA and decreased when higher amounts of N10 pDNA (40 or $80 \mu \mathrm{g}$ ) were applied (Figure 3B).

\section{Influence of the Cell Density}

All the results shown were obtained with exponentially growing cells. When cells were overgrown, the transfection efficiency and cell survival decreased significantly (data not shown). Electroporation was performed at a constant volume of $300 \mu \mathrm{L}$ per cuvette in all experiments. Under the described electroporation conditions $(200 \mathrm{~V}$ and $975 \mu \mathrm{F})$, an increase in volume led to shorter pulse times and less cell death but decreased transfection efficiency.

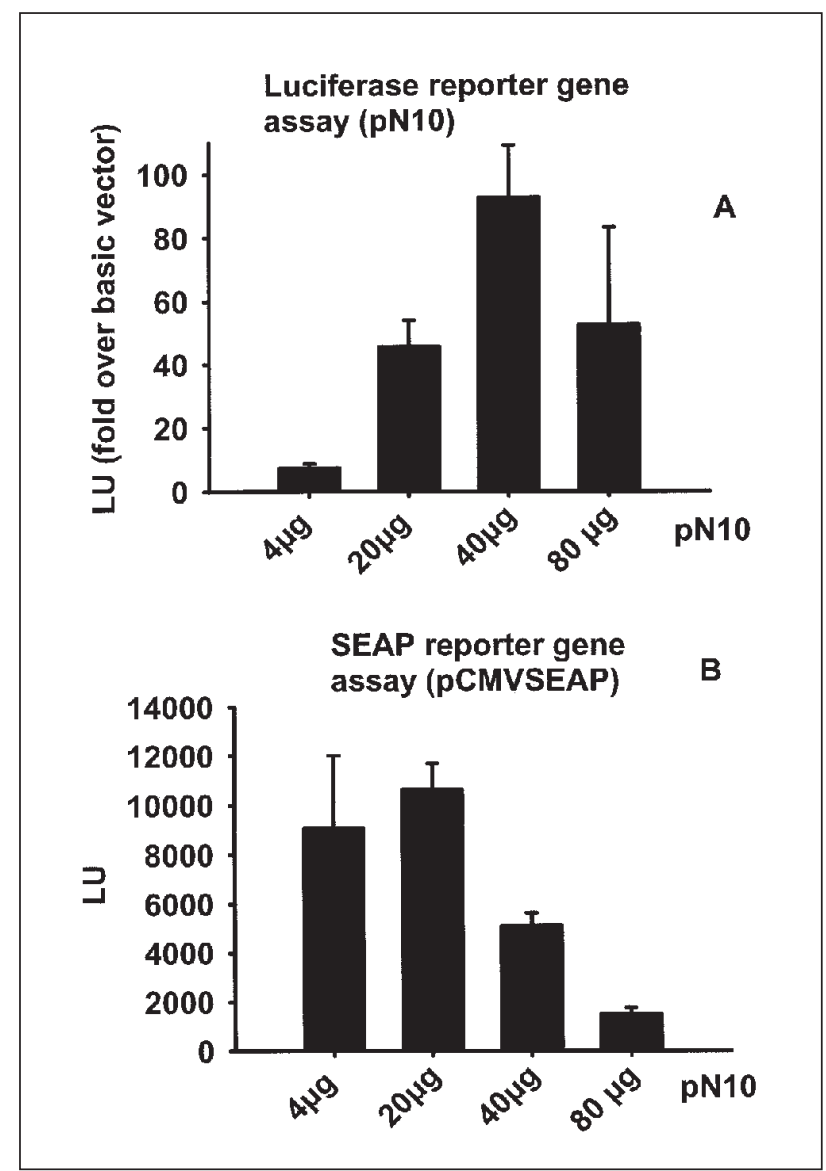

Figure 3. Effect of DNA concentration on reporter gene activity in Mono Mac 6 cells. The indicated amounts of 5-lipoxygenase promoter construct and $1 \mu \mathrm{g}$ pCMVSEAP construct were transfected into Mono Mac 6 cells. Six hours after electroporation, luciferase reporter gene assays (A) and SEAP reporter gene assays (B) were performed, and the results are shown as corrected light units over pGL3Basic vector for the luciferase construct and as corrected light units for SEAP activity. Results are indicated as the mean value of the corrected light units and the SEM of a representative experiment. LU, light units. Transfections were performed in triplicate. ecules to enter or leave the cell $(18,31)$. The pore number and diameter increase with the product of the electric field and pulse duration. Because transfection efficiency sharply peaked at $200 \mathrm{~V}$ in Mono Mac 6 cells and almost no transfection occurred at $150 \mathrm{~V}$ or lower, it seems that a minimal voltage is required to produce pores, which is in accordance with the calculated critical field strength and the resulting minimal voltage of $180 \mathrm{~V}$ under our transfection conditions. Additional increases in the voltage induce pore diameters and total pore areas that are too large for the cell to recover from the transfection, which causes the cells to be irreversibly damaged (Figures 1 and 2). Thus, electroporations of Mono Mac 6 cells should be performed at the optimum voltage $(200 \mathrm{~V})$ according to our protocol.

In addition, an important parameter for cell death appears to be the $\mathrm{pH}$ change that occurs due to electrolysis close to the electrodes. Some protocols recommend alleviating this problem by reducing the pulse length or a part of the ionic strength of the media or PBS with extra buffer such as HEPES or Tris at $\mathrm{pH} 7.5$, whereas empirical studies and our experience (data not shown) have shown that EDTA and buffer salts such as HEPES or Tris can drastically reduce transfection efficiency (1). When we used short pulses of 40-400 $\mu \mathrm{s}$, which lead to low local $\mathrm{pH}$ changes and only small amounts of $\mathrm{Al}^{3+}$ ions that are solubilized from the electrodes are large enough to allow macromol-

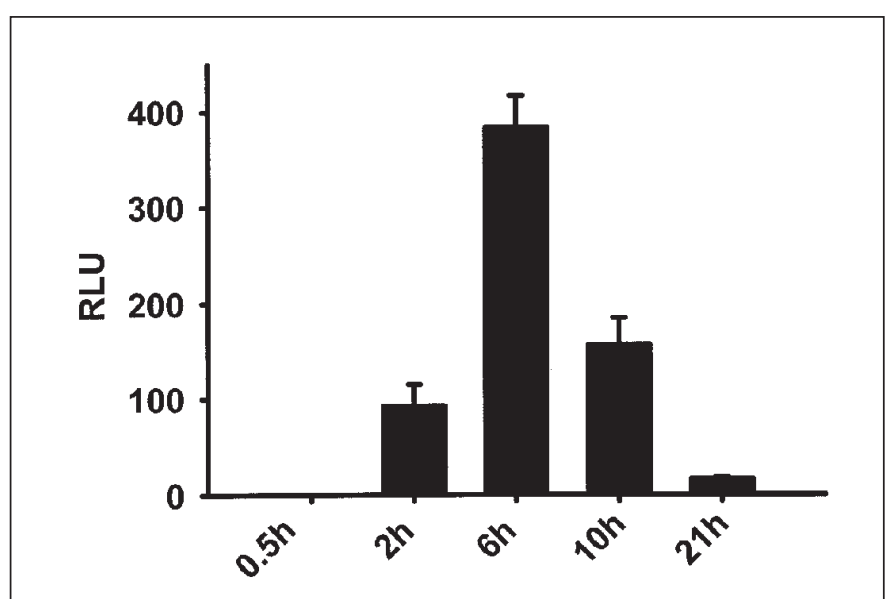

Figure 4. Time course of luciferase activity in Mono Mac 6 cells. Forty micrograms of pN10 and $1 \mu \mathrm{g}$ pCMVSEAP (internal standard) were transfected into Mono Mac 6 cells, and the luciferase activity was determined at the indicated times after transfection. Results are given as the relative light units (SEM of at least three experiments). RLU, relative light units. 
upon pulsing (9), relatively high survival rates were obtained (up to $80 \%$ with $975 \mu \mathrm{F}, 40 \mu \mathrm{s}$, and $200 \mathrm{~V}$ ); however, luciferase activities were similar to mock-transfected cells, indicating a very low transfection efficiency. The data suggest that the pulse length should be in the range of 30-40 ms.

In addition to the conventional single pulse systems, double pulse systems have been recently developed, based on the rationale that the first high-voltage pulse creates pores or openings in the cell membrane because of the high voltage delivered, and the second low-voltage pulse delivers energy to those cells shocked by the first pulse, therefore favoring the entry of exogenous molecules into the cells by creating an active electrophoresis field. While the transient formation of pores in the plasma membrane following the delivery of the appropriate electrical fields has been documented via elec- tron microscopy (5), the mechanism of entry of materials through these pores is not known, and no evidence provides for the postulated mechanism. Experimentally, no benefits were observed in our experiments using the double pulse technique (data not shown), which is in agreement with results from other studies (15).

Finally, in the case of $\mathrm{pN} 10$, a vector to standard quotient of 40:1 may be regarded as safe because the obtained SEAP activities are still in the dynamic range. Nevertheless, in co-transfection studies, the influence of additional expression vectors on the standard plasmid has to be controlled and, if possible, corrected by keeping the total amount of transfected pDNA constant by the addition of mock pDNA. Care should be taken when choosing the mock pDNA, and individual interactions with the internal standard must be excluded.

Because Mono Mac 6 cells grow rapidly in suspension and are extremely easy to culture, they represent an interesting cell system to study the regulation of many myeloid genes. In the case of the 5-lipoxygenase promoter, we obtained reporter gene activities in Mono Mac 6 cells that were approximately 1200 -fold higher than the promoterless control vector pGL3Basic, whereas much lower promoter activity was found in the 5-lipoxygenase-negative cell line HeLa (33-fold). On the other hand, the 5-lipoxygenase promoter was approximately 100-fold less active than the CMV promoter that was used as the positive control.

Our optimized transfection protocol for Mono Mac 6 cells and the sensitivity of the luciferase assay allows for the characterization of promoters with low and cell-specific transcriptional activity, which eliminates the need for stable transfections that are more time consuming and labor intensive. 


\section{ACKNOWLEDGMENTS}

We thank Dagmar Szellas and Astrid Brüggerhoff for expert technical assistance. This study was supported by grants from the European Union (QLG1-CT-2001-01521), the Wilhelm Sander Foundation, and the Fonds der Chemischen Industrie.

\section{REFERENCES}

1.Baum, C., P. Forster, S. Hegewisch-Becker, and K. Harbers. 1994. An optimized electroporation protocol applicable to a wide range of cell lines. BioTechniques 17:1058-1062.

2.Brungs, M., O. Rådmark, B. Samuelsson, and D. Steinhilber. 1994. On the induction of 5-lipoxygenase expression and activity in HL60 cells: effects of vitamin D3, retinoic acid, DMSO and TGF $\beta$. Biochem. Biophys. Res. Commun. 205:1572-1580.

3.Brungs, M., O. Rådmark, B. Samuelsson, and D. Steinhilber. 1995. Sequential induction of 5-lipoxygenase gene expression and activity in Mono Mac 6 cells by transforming growth factor $\beta$ and 1,25- dihydroxyvitamin D3. Proc. Natl. Acad. Sci. USA 92:107-111.

4.Chang, D.C. 1997. Experimental strategies in efficient transfection of mammalian cells. Electroporation. Methods Mol. Biol. 62:307-318.

5.Chang, D.C. and T.S. Reese. 1990. Changes in membrane structure induced by electroporation as revealed by rapid-freezing electron microscopy. Biophys. J. 58:1-12.

6.Chu, G., H. Hayakawa, and P. Berg. 1987. Electroporation for the efficient transfection of mammalian cells with DNA. Nucleic Acids Res. 15:1311-1326.

7.Drazen, J.M., C.N. Yandava, L. Dube, N. Szczerback, R. Hippensteel, A. Pillari, E. Israel, N. Schork, et al. 1999. Pharmacogenetic association between ALOX5 promoter genotype and the response to anti-asthma treatment. Nat. Genet. 22:168-170.

8.Ehlert, F., P. Bierbaum, and J. Schorr. 1993 Importance of DNA quality for transfection efficiency. BioTechniques 14:546.

9.Friedrich, U., N. Stachowitz, A. Simm, G. Fuhr, K. Lucas, and U. Zimmermann. 1998. High efficiency electrotransfection with aluminium electrodes using microsecond controlled pulses. Bioelectrochem. Bioenerg. 47:103-111.

10.Gaunitz, F., M. Papke, and R. Gebhardt. 1996. Transient transfection of primary cultured hepatocytes using CaPO4/DNA precipitation. BioTechniques 20:826-832.

11.Hama-Inaba, H., T. Nishimoto, M. Ohtsubo, K. Sato, and M. Kasai. 1988. Simple and effective method of electroporation for introduction of plasmid and cosmid DNAs to mammalian cells. Nucleic Acids Symp. Ser. 19:149-152.

12.Heiser, W.C. 2000. Optimizing electroporation conditions for the transformation of mammalian cells. Methods Mol. Biol. 130:117-134.

13.Hoshiko, S., O. Rådmark, and B. Samuels- son. 1990. Characterization of the human 5 lipoxygenase gene promoter. Proc. Natl. Acad. Sci. USA 87:9073-9077.

14.In, K.H., K. Asano, D. Beier, J. Grobholz, P.W. Finn, E.K. Silverman, E.S. Silverman, T. Collins, et al. 1997. Naturally occurring mutations in the human 5-lipoxygenase gene promoter that modify transcription factor binding and reporter gene transcription. $\mathrm{J}$. Clin. Invest. 99:1130-1137.

15.Knutson, J.C. and D. Yee. 1987. Electroporation: parameters affecting transfer of DNA into mammalian cells. Anal. Biochem. 164:44-52.

16.Martin, C.S., P.A. Wight, A. Dobretsova, and I. Bronstein. 1996. Dual luminescencebased reporter gene assay for luciferase and $\beta$ galactosidase. BioTechniques 21:520-524.

17.Maxwell, I.H. and F. Maxwell. 1988. Electroporation of mammalian cells with a firefly luciferase expression plasmid: kinetics of transient expression differ markedly among cell types. DNA 7:557-562.

18.Neumann, E., M. Schaefer-Ridder, Y. Wang, and P.H. Hofschneider. 1982. Gene transfer into mouse lyoma cells by electroporation in high electric fields. EMBO J. 1:841-845.

19.Pahl, H.L., T.C. Burn and D.G. Tenen. 1991. Optimization of transient transfection into human myeloid cell lines using a luciferase reporter gene. Exp. Hematol. 19:1038-1041.

20.Pahl, H.L., A.G. Rosmarin, and D.G. Tenen. 1992. Characterization of the myeloid specific CD11b promoter. Blood 79:865-870.

21.Putirka, S. and M. Iacobelli. 1993. Optimizing electroporation of mammalian cells. Am. Biotechnol. Lab. 11:18.

22.Silverman, E.S. and J.M. Drazen. 2000. Genetic variations in the 5-lipoxygenase core promoter. Description and functional implications. Am. J. Respir. Crit. Care Med. 161:S77-S80.

23.Silverman, E.S., J. Du, G.T. De Sanctis, O. Rådmark, B. Samuelsson, J.M. Drazen, and T. Collins. 1998. Egr-1 and Sp1 interact functionally with the 5-lipoxygenase promoter and its naturally occurring mutants. Am. J. Respir. Cell Mol. Biol. 19:316-323.

24.Singal, R. and G.D. Ginder. 1999. DNA methylation. Blood 93:4059-4070.

25.Uhl, J., N. Klan, M. Rose, K.D. Entian, O. Werz, and D. Steinhilber. 2002. The 5lipoxygenase promoter is regulated by DNA methylation. J. Biol. Chem. 277:4374-4379.

26.Wolf, H., M.P. Rols, E. Boldt, E. Neumann, and J. Teissie. 1994. Control by pulse parameters of electric field-mediated gene transfer in mammalian cells. Biophys. J. 66:524-531.

27.Zhang, D.E., C.J. Hetherington, S. Tan, S.E. Dziennis, D.A. Gonzalez, H.M. Chen, and D.G. Tenen. 1994. Sp1 is a critical facto for the monocytic specific expression of human CD14. J. Biol. Chem. 269:11425-11434.

28.Ziegler-Heitbrock, H.W., W. Schraut, P. Wendelgass, M. Strobel, T. Sternsdorf, C. Weber, M. Aepfelbacher, M. Ehlers, et al. 1994. Distinct patterns of differentiation induced in the monocytic cell line Mono Mac 6. J. Leukoc. Biol. 55:73-80.

29.Ziegler-Heitbrock, H.W., E. Thiel, A. Futterer, V. Herzog, A. Wirtz, and G. Riethmuller. 1988. Establishment of a human cell line (Mono Mac 6) with characteristics of mature monocytes. Int. J. Cancer 41:456-461.

30.Zimmermann, U. 1996. The effect of high intensity electric field pulses on eukaryotic cell membranes: fundamentals and application, $\mathrm{p}$. 1. In U. Zimmermann and G.A. Neil (Eds.), Electromanipulation of Cells. CRC Press, Boca Raton, FL.

31.Zimmermann, U., F. Riemann, and G. Pilwat. 1976. Enzyme loading of electrically homogeneous human red blood cell ghosts prepared by dielelectric breakdown. Biochim. Biophys. Acta 436:460-474.

Received 18 June 2002; accepted 18 September 2002

Address correspondence to:

Dr. Dieter Steinhilber

Institute of Pharmaceutical Chemistry

University of Frankfurt

Marie-Curie-Str. 9

60439 Frankfurt, Germany

e-mail: steinhilber@em.uni-frankfurt.de

For reprints of this or any other article, contact Reprints@BioTechniques.com 